\title{
Clinical significance of radiation-induced liver disease after stereotactic body radiation therapy for hepatocellular carcinoma
}

\author{
Baek Gyu Jun ${ }^{1}$, Young Don Kim ${ }^{1}$, Gab Jin Cheon ${ }^{1}$, Eun Seog Kim², Eunjin Jwa², Sang Gyune Kim³, \\ Young Seok Kim³ , Boo Sung Kim³ ${ }^{3}$, Soung Won Jeong ${ }^{4}$, Jae Young Jang ${ }^{4}$, Sae Hwan Lee ${ }^{5}$, and Hong Soo Kim
}

\begin{abstract}
${ }^{1}$ Department of Internal Medicine, Gangneung Asan Hospital, University of Ulsan College of Medicine, Gangneung; ${ }^{2}$ Department of Radiation Oncology, Soonchunhyang University Cheonan Hospital, Cheonan; ${ }^{3}$ Department of Internal Medicine, Soonchunhyang University Bucheon Hospital, Bucheon; ${ }^{4}$ Department of Internal Medicine, Soonchunhyang University Seoul Hospital, Seoul; ${ }^{5}$ Department of Internal Medicine, Soonchunhyang University Cheonan Hospital, Cheonan, Korea
\end{abstract}

\section{Received: December 12, 2016 Revised : February 8, 2017 Accepted: March 17, 2017}

\section{Correspondence to} Hong Soo Kim, M.D. Division of Gastroenterology, Department of Internal Medicine, Soonchunhyang University Cheonan Hospital, 31 Suncheonhyang 6-gil, Dongnam-gu,

Cheonan 31151, Korea

Tel: +82-41-570-3737

Fax: +82-41-570-3728

E-mail: khskhs@schmc.ac.kr
Background/Aims: The aim of this study was to investigate parameters that predict radiation-induced liver disease (RILD) following stereotactic body radiotherapy (SBRT) in patients with hepatocellular carcinoma (HCC) and to identify the clinical significance of RILD.

Methods: We retrospectively reviewed the medical records of 117 HCC patients who were treated by SBRT from March 2011 to February 2015. RILD was defined as elevated liver transaminases more than five times the upper normal limit or a worsening of Child-Pugh (CP) score by 2 within 3 months after SBRT. All patients were assessed at 1 month and every 3 months after SBRT.

Results: Median follow-up was 22.5 months (range, 3 to 56) after SBRT. RILD was developed in 29 of the 117 patients (24.7\%). On univariate analysis, significant predictive factors of RILD were pretreatment CP score $(p<0.001)$ and normal liver volume $(p=0.002)$. Multivariate analysis showed that $\mathrm{CP}$ score was a significant predictor of RILD $(p<0.001)$. The incidence of RILD increased above a CP score of 6 remarkably. The rate of recovery from RILD decreased significantly above a $\mathrm{CP}$ score of 8 . Survival analysis showed that CP score was an independent prognostic factor of overall survival $(p=0.001)$.

Conclusions: CP score is a significant factor to predict RILD in patients with chronic liver disease. RILD can be tolerated by patients with a CP score $\leq 7$. However, careful monitoring of liver function is needed for patients with a CP score 7 after SBRT.

Keywords: Carcinoma, hepatocellular; Stereotactic body radiation therapy; Radiation-induced liver disease; Child-Pugh score

\section{INTRODUCTION}

Hepatocellular carcinoma (HCC) is the third most common cause of cancer-related death worldwide [1]. Surgical resection is the standard treatment for early-stage HCC. However, many cases are unsuitable for resection or ablative treatments because of severe concurrent cir- rhosis or advanced HCC [2]. In such cases, radiotherapy (RT) and transarterial chemoembolization are considered as secondary treatments $[3,4]$. Previously, RT has been infrequently performed due to the relatively low tolerance of the whole liver to RT [5]. Technological advances have made it possible for high doses of radiation to conform to the target volume safely $[6,7]$. Ste- 
reotactic body radiotherapy (SBRT) allows for delivery of a high dose of radiation in a few fractions to HCC by image-guided radiation therapy accompanied by a high degree of accuracy in target delineation. SBRT has been used to treat patients with small HCC who are not eligible for surgery, radiofrequency ablation, or liver transplantation [8].

Radiation-induced liver disease (RILD) is a significant limiting factor in the use of RT because there is no effective treatment for RILD. Predictors of RILD have not been well established yet. Definition and terminology describing RILD or hepatic toxicity resulting from HCC treatment vary among studies [9-12]. Predictive factors for RILD also differ depending on the precise definition used, radiation method, and type of cancer. Some authors have reported that dose-volumetric parameters are important in predicting the development of RILD $[6,13,14]$. However, others emphasized that clinical factors could predict the development of RILD $[7,9,15,16]$.

Most patients with HCC have preexisting cirrhosis or hepatitis, which may significantly increase the risk of RILD [6]. Information about RILD in chronic liver disease patients remains limited and the predictive factors for RILD have not been validated, particularly for SBRT.

Therefore, the aim of our study is to identify factors that could be used to predict RILD in patients with HCC who were treated with SBRT and to evaluate the clinical significance of RILD.

\section{METHODS}

\section{Patients}

Patients who were treated with SBRT for primary HCC were registered in this study. Data collected between March 2011 and February 2015 in Soonchunhyang University Cheonan Hospital were retrospectively reviewed. The inclusion criteria were as follows: primary HCC, pretreatment Child-Pugh (CP) class A or B, HCC with no evidence of major vascular invasion, no evidence of extrahepatic spread and follow-up time $\geq 3$ months. One hundred and thirty-nine patients were registered in this study. Of these 139 patients, 22 were excluded because they had double primary cancer (four patients), follow-up time < 3 months (six patients), major vascular invasion (nine patients), or extrahepatic spread (three pa- tients). The remaining 117 patients were finally enrolled. This study was approved by the Institutional Review Board of Soonchunhyang University Cheonan Hospital (IRB number: 2015-08-013-001). This study was approved by the Institutional Review Board of Soonchunhyang University Cheonan Hospital (IRB number: 2015-08-013o०1) and written informed consent was waived because of the retrospective study.

\section{SBRT procedure}

During the four-dimensional $(4 \mathrm{D})$ computed tomography $(\mathrm{CT})$ simulation, patients were immobilized in supine position with arms above the head. Respiration-correlated $4 \mathrm{D}$ CT scans were performed during quiet free breathing using a real-time position management system (Varian Medical Systems, Palo Alto, CA, USA) and a 16-slice CT scanner (Brilliance CT Big Bore, Philips Medical Systems, Cleveland, OH, USA) with slice thickness of $0.2 \mathrm{~cm}$. CT series were sorted according to respiratory phase from o to 90 , each of which reflecting $10 \%$ of the respiratory cycle.

Gross tumor volume (GTV) was measured based on visible gross tumor as seen on CT images at the end-expiratory phase fused with multiphase MR images. Extension based on movement within the gating phase (30\% to $70 \%$ ) from the GTV was set as the internal target volume (ITV). The planning target volume (PTV) was defined as the volume with a $5 \mathrm{~mm}$ margin added to the ITV. SBRT was planned using coplanar and/or non-coplanar photon beams with energies of 6 or $15 \mathrm{MV}$ (Eclipse V8.9, Varian Medical Systems). Total dose administered was 40 to 60 Gy (median, 55) at the PTV in three to five fractions over consecutive days or twice a week. The chosen isodose covering PTV was between 85 to $95 \%$, which was normalized to the center of the PTV.

Treatment was delivered using a Novalis Tx system equipped with an On-Board Imager (OBI; Varian Medical Systems) and a highdefinition multileaf collimator consisting of 120 leaves including $2.5 \mathrm{~mm}$ central and $5 \mathrm{~mm}$ peripheral leaves (Varian Medical Systems; and BrainLab, Feldkirchen, Germany). Image guidance with cone beam CT was performed prior to the administration of each fraction of SBRT using the OBI.

\section{Definition and evaluation of RILD}

RILD was defined as elevated liver transaminases more 
than five times the upper normal limit or worsening of CP score by 2 or more within 3 months after SBRT [17]. CP score was confirmed on two consecutive visits at least 1 month apart before RT [18]. Recovery from RILD was defined as decrease of CP score or normalization of hepatic enzymes to pre-SBRT level within 6 months after development of RILD. Hepatitis B virus (HBV) reactivation and worsening liver function related to other causes was excluded from RILD.

The clinical parameters analyzed were gender, age, CP score, HBV, aspartate aminotransferase (AST), alanine aminotransferase (ALT), and alcohol use. Dose-volumetric parameters analyzed were normal liver volume (NLV), GTV, PTV, SBRT dose, dose per fraction, and $\mathrm{NLV}$ after receiving $>5\left(\mathrm{~V}_{5}\right), 10\left(\mathrm{~V}_{10}\right), 15\left(\mathrm{~V}_{15}\right), 20\left(\mathrm{~V}_{20}\right), 25$ $\left(\mathrm{V}_{25}\right), 30\left(\mathrm{~V}_{30}\right), 35\left(\mathrm{~V}_{35}\right), 40\left(\mathrm{~V}_{40}\right), 45\left(\mathrm{~V}_{45}\right), 50\left(\mathrm{~V}_{50}\right), 55\left(\mathrm{~V}_{55}\right)$, or $60 \mathrm{~Gy}\left(\mathrm{~V}_{60}\right)$ radiation therapy. NLV was the total liver volume (TLV) minus the GTV of HCC.

All patients were examined during SBRT to assess acute toxicity. After treatment, the patients were followed up every 1 to 3 months. Physical examinations, complete blood counts, biochemical profiles, tumor markers, and three-phasic CT scan or magnetic resonance imaging scan for liver were performed at every follow-up visit.

\section{Statistical analysis}

Chi-square, Fisher exact test, or Student $t$ test were performed for univariate analysis of an association between different variables and the risk of RILD. One-way analysis of variance was used for comparing more than three variables. In multivariate analysis, binary logistic regression model was constructed with all parameters that were significant in univariate analysis. A linear by linear association model was used to identify the probability of RILD according to increase in CP score. Cox proportional hazards regression analysis was used to predict the risk factor for survival. A $p<0.05$ was considered significant. All statistical analyses were performed using SPSS version 18.0 (SPSS Inc., Chicago, IL, USA).

\section{RESULTS}

\section{Patient characteristics}

One hundred and seventeen patients were evaluated.
The median follow-up period after completion of SBRT was 22.5 months (range, 3 to 56). RILD was observed in 29 of the 117 patients (24.7\%) after SBRT. Worsening of CP score by 2 was observed in 26 of these 29 patients (89.6\%). Elevation of liver transaminases to more than five times the upper normal limit was observed in three of the 29 patients (10.4\%). The most common etiologies of HCC were HBV (55.5\%) and alcohol use (27.3\%). Baseline characteristics of these patients are summarized in Table 1.

\section{Variables for the risk of RILD}

In the univariate analysis, significant predictive factors of RILD included pretreatment CP score $(p<0.001)$ and NLV $(p=0.002)$. Age, gender, HBV, alcohol use, AST, ALT, SBRT dose, dose per fraction, GTV, and PTV did not influence the possibility of RILD. Dosimetric characteristics $\left(\mathrm{V}_{5}, \mathrm{~V}_{10}, \mathrm{~V}_{15}, \mathrm{~V}_{20}, \mathrm{~V}_{25}, \mathrm{~V}_{30}, \mathrm{~V}_{35}, \mathrm{~V}_{40}, \mathrm{~V}_{45}, \mathrm{~V}_{50}, \mathrm{~V}_{55}\right.$, and $V_{60}$ ) showed no correlation with RILD (Table 2). In multivariate logistic regression analysis, only pretreatment CP score was found to be significantly associated with RILD ( $p<0.001)$. More RILD cases occurred as CP score increased (CP score 6 [CP-A6]: odds ratio [OR], 7.615; $95 \%$ confidence interval [CI], 1.882 to 30.819; $p=$ 0.004) (CP score 7 [CP-B7]: OR, 16.500; 95\% CI, 3.852 to 70.672; $p<0.001$ ) (CP score $\geq 8$ [CP $\geq \mathrm{B} 8]$ : OR, 115.500; 95\% CI, 13.090 to $3,020.514 ; p<0.001$ ) (Table 3).

\section{Relationship between RILD and CP score}

According to the linear by linear association model, the probability of RILD was positively correlated with an increase in pretreatment CP score $(p<0.001)$. The incidence of RILD after SBRT was $5.7 \%$ (four of 70 patients) in patients with a CP score of 5 (CP-A5), 31.6\% (six of 19 patients) in patients with $\mathrm{CP}-\mathrm{A6}, 50.0 \%$ (seven of 14 patients) in patients with $\mathrm{CP}-\mathrm{B} 7,83.3 \%$ (10 of 12 patients) in patients with CP score 8 (CP-B8), and 100\% (two of two patients) in patients with $\mathrm{CP}$ score 9 (CP-B9). The incidence of RILD increased remarkably above CP-A6. The recovery rate from RILD also decreased as pretreatment CP score increased $(p<0.001)$. The recovery rate from RILD was $100 \%$ (four of four patients) in patients with CP-A5, 50.0\% (three of six patients) in patients with CP-A6, 42.9\% (three of seven patients) in patients with $\mathrm{CP}-\mathrm{B} 7, \mathrm{O} \%$ (o of $10 \mathrm{pa-}$ tients) in patients with CP-B8, and o\% (o of two patients) in patients with $\mathrm{CP}-\mathrm{B}_{9}$. The recovery rate from RILD decreased significantly above CP-B8 (Fig. 1). 
Table 1. Baseline characteristics of the study subjects

\begin{tabular}{|c|c|}
\hline Characteristic & Value \\
\hline No. of patients & 117 \\
\hline Sex, male/female & $86 / 31$ \\
\hline Age, yr & $63(38-85)$ \\
\hline Tumor size, $\mathrm{cm}$ & $2.1(1.0-4)$ \\
\hline \multicolumn{2}{|l|}{ No. of lesions for patient } \\
\hline 1 & $99(84.6)$ \\
\hline 2 & $17(14 \cdot 5)$ \\
\hline 3 & $1(0.9)$ \\
\hline \multicolumn{2}{|l|}{ Child-Pugh class } \\
\hline A & $89(76.1)$ \\
\hline $\mathrm{B}$ & $28(23.9)$ \\
\hline \multicolumn{2}{|l|}{ Child-Pugh score } \\
\hline 5 & $73(62.3)$ \\
\hline 6 & $16(13.7)$ \\
\hline 7 & $14(12.0)$ \\
\hline 8 & $12(10.3)$ \\
\hline 9 & $2(1.7)$ \\
\hline \multicolumn{2}{|l|}{ BCLC stage } \\
\hline A & $21(17 \cdot 9)$ \\
\hline $\mathrm{B}$ & $96(82.1)$ \\
\hline \multicolumn{2}{|l|}{ Hepatitis etiology } \\
\hline Alcohol & $32(27 \cdot 4)$ \\
\hline Hepatitis B virus & $65(55 \cdot 5)$ \\
\hline Hepatitis $\mathrm{C}$ virus & $11(9.4)$ \\
\hline Others & $9(7 \cdot 7)$ \\
\hline \multicolumn{2}{|l|}{ Prior treatment } \\
\hline Transarterial chemoembolization & $76(64.9)$ \\
\hline Radiofrequency ablation & $24(20.5)$ \\
\hline Surgery & $1(0.9)$ \\
\hline Radiotherapy & $16(13.7)$ \\
\hline Radiation dose, Gy & $54.7 \pm 5.84$ \\
\hline Fraction & $3(3-5)$ \\
\hline Dose per fraction, Gy & $16.7 \pm 0.32$ \\
\hline $\mathrm{NLV}, \mathrm{cm}^{3}$ & $1,176.12 \pm 342.25$ \\
\hline GTV, $\mathrm{cm}^{3}$ & $8.10 \pm 5.10$ \\
\hline PTV, $\mathrm{cm}^{3}$ & $38.76 \pm 19.08$ \\
\hline
\end{tabular}

Values are presented as median (range), number (\%), or mean $\pm \mathrm{SD}$.

BCLC, Barcelona Clinic Liver Cancer; NLV, normal liver volume; GTV, gross tumor volume; PTV, planning target volume.

\section{Change in liver function after SBRT}

We evaluated changes in CP score during 6 months after SBRT. Results are shown in Fig. 2. Of the 117 patients, 10 patients were excluded because another treatment for HCC was performed within 6 months after SBRT. Change in CP score after SBRT became larger with an increase in the CP score $(p<0.001)$. The change in CP score was $0.27,0.68,1.29,2.2$, and 2.5 points in patients with CP-A5, CP-A6, CP-B7, CP-B8, and CP-B9 at 3 months, respectively. Changes in $\mathrm{CP}$ score in patients with $\mathrm{CP}$ A5 and CP-A6 were less than 1 point. The CP score was improved slightly at 6 months after $\operatorname{SBRT}$ (5.27 to 5.22 in patients with CP-A5 and 6.68 to 6.53 in patients with $\mathrm{CP}$ A6). In patients with $\mathrm{CP}-\mathrm{B} 7$ and $\mathrm{CP}-\mathrm{B} 8$, no improvement of CP score was observed at 6 months after SBRT (8.29 to 8.36 in patients with $\mathrm{CP}-\mathrm{B} 7$ and 10.2 to 10.33 in patients with CP-B8). In two patients with CP-B9, the CP score at 6 months could not be evaluated because both of them died of hepatic failure.

\section{Relationship between RILD and survival}

In the Cox proportional hazard model, sex, presence of RILD, CP score, dose per fraction, and NLV had prognostic significance for survival. Prognostic factors identified by univariate analysis were analyzed by multivariate analysis. Only CP score was an independent prognostic factor affecting the overall survival on multivariate analysis (CP-A6: hazard ratio (HR), 0.942; 95\% CI, 0.513 to $1.729 ; p=0.847$ ) (CP-B7: HR, 1.578; 95\% CI, o.770 to 3.236; $p=0.001)(\mathrm{CP} \geq \mathrm{B} 8$ : HR, 5.161; 95\% CI, 1.986 to 13.409; $p=0.005$ ) (Table 4). Of 117 patients, a total of 30 died during the follow-up period. Patients who died of hepatic failure due to RILD were analyzed. Two of the 29 RILD patients (6.8\%) died of hepatic failure within 6 months after the onset of RILD. Death related to RILD occurred only in patients with CP-B9.

\section{DISCUSSION}

SBRT has been critical in improving local control or as a potential cure in patients with small HCC as an alternative treatment [8]. Although SBRT has a good local control rate, the risk of RILD still remains uncertain. RILD has long been reported as one of the most serious treatment-related complications for patients who 
Table 2. Univariate analysis of parameters associated with the risk of RILD

\begin{tabular}{|c|c|c|c|}
\hline Variable & $\operatorname{RILD}(n=29)$ & Non-RILD $(\mathrm{n}=88)$ & $p$ value \\
\hline Sex & & & 0.803 \\
\hline Male & $23(25.9)$ & $66(74.1)$ & \\
\hline Female & $6(21.4)$ & $22(87.6)$ & \\
\hline Age, yr & $61(38-79)$ & $62(38-80)$ & 0.588 \\
\hline Child-Pugh score & & & $<0.001$ \\
\hline 5 & $4(5.7)$ & $66(94.3)$ & \\
\hline 6 & $6(31.6)$ & $13(68.4)$ & \\
\hline 7 & $7(50.0)$ & $7(50.0)$ & \\
\hline 8 & $10(83.3)$ & $2(16.7)$ & \\
\hline 9 & $2(100)$ & 0 & \\
\hline \multicolumn{4}{|l|}{ Hepatitis etiology } \\
\hline Alcohol & & & 0.320 \\
\hline Yes & $10(31.2)$ & $22(68.8)$ & \\
\hline No & $19(22.3)$ & $66(77.7)$ & \\
\hline Hepatitis B virus & & & 0.363 \\
\hline Yes & $14(21.5)$ & $51(78.5)$ & \\
\hline No & $15(28.8)$ & $37(71.2)$ & \\
\hline AST, IU/L & $37.1 \pm 28.3$ & $36.3 \pm 21.1$ & 0.112 \\
\hline ALT, IU/L & $32.2 \pm 19.8$ & $31.2 \pm 26.0$ & 0.845 \\
\hline SBRT dose, Gy & $56.7 \pm 13.1$ & $54.8 \pm 5.8$ & 0.523 \\
\hline Dose per fraction, Gy & $16.6 \pm 3.84$ & $16.7 \pm 3.17$ & 0.834 \\
\hline $\mathrm{NLV}, \mathrm{cm}^{3}$ & $971.23 \pm 297.90$ & $1,236.01 \pm 332.93$ & 0.002 \\
\hline GTV, $\mathrm{cm}^{3}$ & $8.27 \pm 7.97$ & $7.56 \pm 9.14$ & 0.384 \\
\hline PTV, $\mathrm{cm}^{3}$ & $35.05 \pm 18.62$ & $31.30 \pm 20.09$ & 0.239 \\
\hline $\mathrm{V}_{5}^{\mathrm{a}}$ & $364.9 \pm 213.3$ & $394.4 \pm 216.6$ & 0.621 \\
\hline $\mathrm{V}_{10}$ & $234.6 \pm 154.8$ & $260.4 \pm 177.0$ & 0.561 \\
\hline $\mathrm{V}_{15}$ & $158.4 \pm 115.6$ & $183.2 \pm 127.9$ & 0.454 \\
\hline $\mathrm{V}_{20}$ & $100.3 \pm 71.8$ & $127.8 \pm 94 \cdot 3$ & 0.199 \\
\hline $\mathrm{V}_{25}$ & $67.1 \pm 45.9$ & $74.8 \pm 64$ & 0.584 \\
\hline $\mathrm{V}_{30}$ & $48.3 \pm 32.7$ & $60.8 \pm 41.7$ & 0.199 \\
\hline $\mathrm{V}_{35}$ & $36.1 \pm 24.1$ & $47.2 \pm 32.1$ & 0.126 \\
\hline $\mathrm{V}_{40}$ & $28.0 \pm 19.0$ & $37.4 \pm 24.9$ & 0.103 \\
\hline $\mathrm{V}_{45}$ & $20.4 \pm 15.6$ & $28.3 \pm 21.2$ & 0.098 \\
\hline $\mathrm{V}_{50}$ & $14.2 \pm 12.1$ & $20.3 \pm 17.5$ & 0.204 \\
\hline $\mathrm{V}_{55}$ & $7.6 \pm 8.2$ & $8.2 \pm 11.4$ & 0.578 \\
\hline $\mathrm{V}_{60}$ & $3.3 \pm 5.0$ & $3.5 \pm 8.4$ & 0.941 \\
\hline
\end{tabular}

Values are presented as number (\%), median (range), or mean \pm SD.

RILD, radiation-induced liver disease; AST, aspartate aminotransferase; ALT, alanine aminotransferase; SBRT, stereotactic body radiotherapy; NLV, normal liver volume; GTV, gross tumor volume; PTV, planning target volume.

${ }^{a} \mathrm{~V}_{5}$ is the normal liver volume after receiving $>5$ Gy radiation. In other words, the number in the suffix represents the dose of radiation received. 
Table 3. Multivariate analysis of parameters associated with the risk of radiation-induced liver disease

\begin{tabular}{lccr}
\hline Variable & OR & $95 \%$ CI & $p$ value \\
\hline Child-Pugh score & & & $<.001$ \\
5 & Reference & - & 0.004 \\
6 & 7.615 & $1.882-30.819$ & $<0.001$ \\
7 & 16.500 & $3.852-70.672$ & 0.001 \\
$\geq 8$ & 115.500 & $13.090-3,020.514$ & 0.968 \\
NLV & 1.000 & $0.997-1.003$ & \\
\hline
\end{tabular}

OR, odds ratio; CI, confidence interval; NLV, normal liver volume.

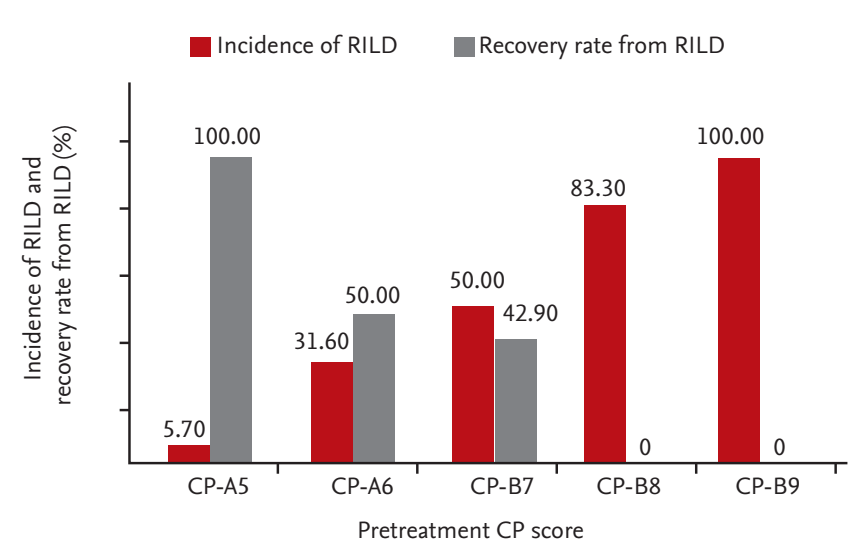

Figure 1. Probability of radiation-induced liver disease (RILD) and recovery from RILD according to Child-Pugh (CP) score. The probability of RILD gradually increased and the probability of recovery from RILD decreased along with an increase in CP score $(p<0.001)$.

undergo hepatic irradiation [5]. However, predictors of RILD have not been well established yet. The precise definition of RILD in patients with HCC who have underlying liver disease varies somewhat between studies. Recently, RILD has been divided into "classic" and "non-classic" types [17]. Classic RILD is subacute hepatic toxicity that presents with anicteric ascites, hepatomegaly, and elevated alkaline phosphatase levels; it typically occurs between 4 and 8 weeks after the completion of RT. Non-classic RILD is defined as elevated liver transaminases more than five times the upper normal limit or worsening of CP score by 2 or more. In our study, RILD was defined according to the non-classic criteria because non-classic RILD was found in patients with underlying chronic liver disease such as cirrhosis or viral hepatitis [19]. In this study, RILD occurred in 29 of

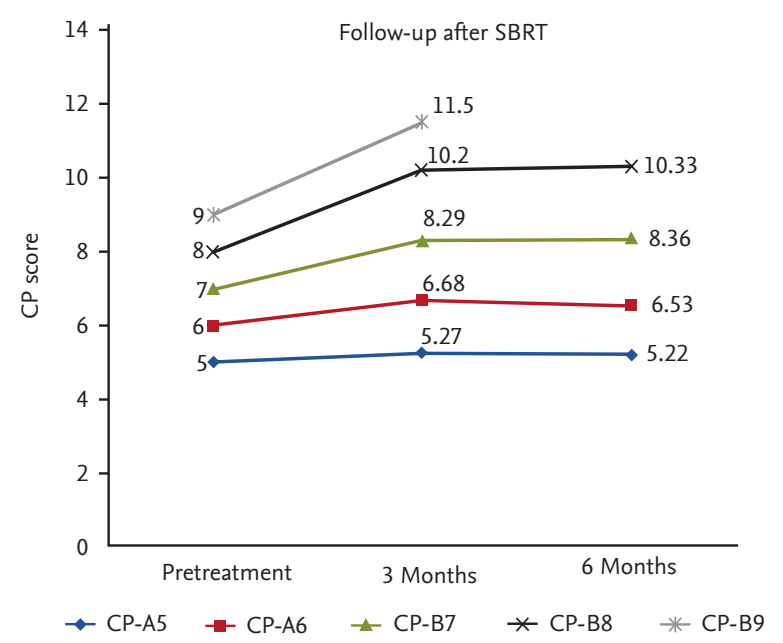

Figure 2. Change in Child-Pugh (CP) score within 6 months after stereotactic body radiotherapy (SBRT). The change in CP score at 3 months after SBRT gradually increased along with an increase in the pretreatment CP score (one-way analysis of variance, $p<0.001)$. The change in CP score was $0.27,0.68,1.29,2.2$, and 2.5 at a CP score of $5,6,7,8$, and 9 at 3 months, respectively.

the 117 patients. However, classic RILD did not occur at all. These results were consistent with those of previous studies showing that non-classic RILD was observed in patients with chronic liver disease [13,20].

Our results showed that only pretreatment CP score was significantly associated with the development of RILD. Previous studies have also reported that pretreatment liver function was an important factor in predicting RILD [9,16]. According to Jung et al. [9], the risk of liver toxicity was high in cases of CP class B. Cardenes et al. [16] have reported that CP score is the only factor related to liver toxicity greater than grade 3 . The severity of liver cirrhosis is an important clinical predictor of RILD. 
Table 4. Analysis of overall survival using the Cox model

\begin{tabular}{|c|c|c|c|c|c|c|}
\hline \multirow{2}{*}{ Variable } & \multicolumn{3}{|c|}{ Univariate analysis } & \multicolumn{3}{|c|}{ Multivariate analysis } \\
\hline & HR & $95 \% \mathrm{CI}$ & $p$ value & HR & $95 \% \mathrm{CI}$ & $p$ value \\
\hline RILD & $5 \cdot 732$ & $2.299-14.295$ & $<0.001$ & 0.980 & $0.299-3.125$ & 0.573 \\
\hline Sex, male/female & 0.211 & $0.050-0.887$ & 0.034 & 0.257 & $0.057-1.156$ & 0.077 \\
\hline Age & 1.023 & $0.978-1.070$ & 0.327 & & & \\
\hline Child-Pugh score & & & & & & 0.001 \\
\hline 5 & Reference & - & - & - & - & - \\
\hline 6 & 0.587 & $0.074-4.653$ & 0.614 & 0.942 & $0.513-1.729$ & 0.847 \\
\hline 7 & 2.531 & $0.526-12.168$ & 0.246 & 1.578 & $0.770-3.236$ & 0.001 \\
\hline$\geq 8$ & 10.647 & $3.695-30.685$ & $<0.001$ & 5.161 & $1.986-13.409$ & 0.005 \\
\hline Hepatitis B virus, yes/no & 0.717 & $0.298-1.726$ & $0.45^{8}$ & & & \\
\hline Alcohol, yes/no & 0.933 & $0.423-2.059$ & 0.863 & & & \\
\hline BCLC stage, A/B & 0.033 & $0.000-3.238$ & 0.145 & & & \\
\hline $\mathrm{AST}, \mathrm{IU} / \mathrm{L}$ & 1.012 & $1.003-1.021$ & 0.108 & & & \\
\hline ALT, IU/L & 1.012 & $1.001-1.023$ & 0.133 & & & \\
\hline SBRT dose, Gy & 0.999 & $0.999-1.000$ & 0.051 & & & \\
\hline Dose per fraction, > 15/ $\leq 15 \mathrm{~Gy}$ & 1.851 & $1.160-2.954$ & 0.010 & 1.245 & $0.581-1.347$ & 0.568 \\
\hline $\mathrm{NLV}, \mathrm{cm}^{3}$ & 0.998 & $0.996-1.000$ & 0.036 & 0.999 & $0.998-1.001$ & 0.493 \\
\hline SBRT response, yes/no & 0.491 & $0.147-1.643$ & 0.248 & & & \\
\hline GTV, $\mathrm{cm}^{3}$ & 1.020 & $0.969-1.075$ & 0.477 & & & \\
\hline PTV, $\mathrm{cm}^{3}$ & 1.010 & $0.989-1.031$ & 0.360 & & & \\
\hline
\end{tabular}

HR, hazard ratio; CI, confidence interval; RILD, radiation-induced liver disease; BCLC, Barcelona Clinic Liver Cancer; AST, aspartate aminotransferase; ALT, alanine aminotransferase; SBRT, stereotactic body radiotherapy; NLV, normal liver volume; GTV, gross tumor volume; PTV, planning target volume.

Prior published reports have shown that patients with CP class B have a high risk of developing $\operatorname{RILD}[9,15,21]$. However, patients with CP-B7, CP-B8, and CP-B9 would not have the same liver function although all of them are in CP class B. In our study, we analyzed the probability of RILD with an increase in CP score. The probability of RILD was positively correlated with an increase in CP score $(p<0.001)$ (Fig. 1). Moreover, RILD developed more often in $\mathrm{CP}-\mathrm{A} 6$ patients than in CP-A5 patients (31.6\% vs. 5.7\%, $p<0.001$ ) (Table 2). This suggests that it is necessary to distinguish CP-A5 and CP-A6 from the perspective of RILD development. Previous studies have shown that CP-A6 patients have worse liver function due to inflammation and fibrosis [22] and poor overall survival [23] compared to CP-A5 patients. Therefore, patients with CP-A6 have high risk of developing RILD compared to patients with CP-A5.

We also examined the recovery rate from RILD after
SBRT with increased the CP score. Only CP-A5 patients recovered completely from RILD. The recovery rate from RILD was moderate in patients with CP-A6 (50\%) and $\mathrm{CP}-\mathrm{B} 7$ (42.9\%). However, the recovery rate from RILD was significantly lower in $\mathrm{CP} \geq \mathrm{B} 8$ patients: $\% \%$ in CP-B8 patients and in CP-B9 patients (Fig. 1). We concluded that RILD was not tolerable in $\mathrm{CP} \geq \mathrm{B}$ 8 patients because the risk of RILD was high and the recovery rate from RILD was low. Several studies have reported that liver toxicity is not tolerable in $\mathrm{CP} \geq \mathrm{B} 8$ patients. Huertas et al. [24] have suggested that liver toxicity is moderate in $\mathrm{CP}$ score $\leq 7(\mathrm{CP} \leq \mathrm{B} 7)$ patients and that only these patients should be deemed eligible for SBRT. A prospective study has suggested that the only factor associated with more than grade 3 or greater liver toxicity or death within 6 months was CP score 8 or greater $(p=0.030)$ [16]. Andolino et al. [25] also have suggested that previous liver function $(\mathrm{CP} \geq \mathrm{B} 8)$ is a limiting factor for toxicity. 
We could predict that SBRT would influence the liver function. However, there are few studies on how liver function changes after SBRT. Tse et al. [26] have suggested that the most clinically significant change after SBRT is a downward change in CP classification from A to B in patients with HCC. In this study, we evaluated the change in liver function at 3 and 6 months after SBRT and found that the decline in liver function was significant in $\mathrm{CP} \geq \mathrm{B} 7$ patients. Changes in liver function in patients with $\mathrm{CP}-\mathrm{A} 5$ and $\mathrm{CP}-\mathrm{A} 6$ were very minimal. Their liver function was improved at 6 months after SBRT. In patients with $\mathrm{CP}-\mathrm{B} 7$, change in the $\mathrm{CP}$ score was more than 1 point. However, CP class remained CP class B without downward change. Especially, deterioration of liver function was severe in patients with $\mathrm{CP} \geq \mathrm{B}$. Their CP class was progressed from CP class B to class $\mathrm{C}$ (Fig. 2). These results suggest that patients with $\mathrm{CP}-\mathrm{A}_{5}$ and $\mathrm{CP}-\mathrm{A} 6$ could tolerate the damage caused by SBRT. In patients with $\mathrm{CP}-\mathrm{B} 7$, there is a need to monitor liver function carefully and adjust dose per fraction and total dose. According to Fig. 1 and 2, it would be appropriate to consider that hepatic reserve function significantly varies from $\mathrm{CP}-\mathrm{A}_{5}$ to $\mathrm{CP}-\mathrm{B}$ 9.

In several previously reported studies, RILD has been associated with dose-volumetric factors. Son et al. [13] have reported that TLV receiving a dose $<18$ Gy is a predictive factor for RILD, which was defined as CP progression or grade 2 or higher hepatic toxicity. The study of Liang et al. [14] has found that the percentage of TLV exceeding 20 Gy irradiation $\left(\mathrm{V}_{20}\right)$ is an independent dose-volume histogram predictor for RILD after SBRT. Another study also has reported that $V_{30}$ is correlated with the occurrence of radiation-induced hepatic toxicity after three-dimensional conformal RT [27]. However, our study showed that dose-volumetric parameters, such as $\mathrm{V}_{20}$ and $\mathrm{V}_{30}$, could not predict RILD after SBRT. The mean NLV was smaller in the patients who had RILD than in those who did not have RILD $\left(971.23 \pm 297.90 \mathrm{~cm}^{3}\right.$ vs. $1,236.01 \pm 332.93 \mathrm{~cm}^{3}, p=0.002$ ). In multivariate analysis, NLV did not predict RILD. Results of study indicated that clinical factors were more influential than dose-volumetric factors in the development of RILD because most patients had chronic liver disease such as viral hepatitis and liver cirrhosis with relatively small irradiated target volume (mean PTV, $38.76 \pm 19.08 \mathrm{~cm}^{3}$ ).

The relationship between RILD and survival has not been validated. A previous study has reported that RILD after SBRT could influence survival. Liang et al. [14] and Son et al. [28] have reported that the presence of RILD can shorten the median survival time. However, in our study, the presence of RILD was not relevant to overall survival. Only CP score was significantly related to overall survival. Although the presence of RILD was not relevant to overall survival, the development of RILD in patients with poor liver function $(\mathrm{CP} \geq \mathrm{B} 8)$ appeared to be a clinically significant factor that could affect overall survival because most $\mathrm{CP} \geq \mathrm{B} 8$ patients did not recover from deteriorated liver function caused by RILD. There have been reports that patients with poor liver function could affect survival. Cardenes et al. [16] have reported that patients with CP-B with scores of 8 or greater are at high risk of severe toxicity and even death. In other study, patients with CP-B7 had improved survival and less toxicity than $\mathrm{CP} \geq \mathrm{B} 8$ patients [29]. According to these results, RILD in $\mathrm{CP} \geq \mathrm{B} 8$ patients could lead to irreversible deterioration of liver function, thereby affecting survival.

Our study has some limitations. First, it was a retrospective study. Second, the study population was relatively small. However, we included only HCC patients treated by SBRT. Third, we included a small number of patients with CP class B. Further investigation is needed to clarify the relationship between RILD and survival.

In conclusion, CP score is closely associated with development of RILD in patients with chronic liver disease. SBRT is a tolerable treatment for patients with $\mathrm{CP} \leq \mathrm{B} 7$, but not in those with $\mathrm{CP} \geq \mathrm{B} 8$. Caution should be used when SBRT is performed for patients with advanced liver cirrhosis.

\section{KEY MESSAGE}

1. Pretreatment Child-Pugh (CP) score was important factor to predict radiation-induced liver disease (RILD) after stereotactic body radiotherapy (SBRT). The increased risk of RILD associated with increased CP score.

2. In patients with CP score $\geq 8$, RILD could lead to significant deterioration of liver function.

3. RILD after SBRT can be tolerable in patients with CP score $\leq 7$. 


\section{Conflict of interest}

No potential conflict of interest relevant to this article was reported.

\section{REFERENCES}

1. Ferlay J, Shin HR, Bray F, Forman D, Mathers C, Parkin DM. Estimates of worldwide burden of cancer in 2008: GLOBOCAN 2008. Int J Cancer 2010;127:2893-2917.

2. Ikai I, Kudo M, Arii S, et al. Report of the 18th follow-up survey of primary liver cancer in Japan. Hepatol Res 2010;40:1043-1059.

3. Sheu JC, Huang GT, Chen DS, et al. Small hepatocellular carcinoma: intratumor ethanol treatment using new needle and guidance systems. Radiology 1987;163:43-48.

4. Ikeda K, Kumada H, Saitoh S, Arase Y, Chayama K. Effect of repeated transcatheter arterial embolization on the survival time in patients with hepatocellular carcinoma: an analysis by the Cox proportional hazard model. Cancer 1991;68:2150-2154.

5. Lawrence TS, Robertson JM, Anscher MS, Jirtle RL, Ensminger WD, Fajardo LF. Hepatic toxicity resulting from cancer treatment. Int J Radiat Oncol Biol Phys 1995;31:1237-1248.

6. Dawson LA, Normolle D, Balter JM, McGinn CJ, Lawrence TS, Ten Haken RK. Analysis of radiation-induced liver disease using the Lyman NTCP model. Int J Radiat Oncol Biol Phys 2002;53:810-821.

7. Liang SX, Zhu XD, Xu ZY, et al. Radiation-induced liver disease in three-dimensional conformal radiation therapy for primary liver carcinoma: the risk factors and hepatic radiation tolerance. Int J Radiat Oncol Biol Phys 2006;65:426-434.

8. Yoon SM, Lim YS, Park MJ, et al. Stereotactic body radiation therapy as an alternative treatment for small hepatocellular carcinoma. PLoS One 2013;8:e79854.

9. Jung J, Yoon SM, Kim SY, et al. Radiation-induced liver disease after stereotactic body radiotherapy for small hepatocellular carcinoma: clinical and dose-volumetric parameters. Radiat Oncol 2013;8:249.

10. Osmundson EC, Wu Y, Luxton G, Bazan JG, Koong AC, Chang DT. Predictors of toxicity associated with stereotactic body radiation therapy to the central hepatobiliary tract. Int J Radiat Oncol Biol Phys 2015;91:986-994.

11. Sanuki N, Takeda A, Oku Y, et al. Influence of liver toxici- ties on prognosis after stereotactic body radiation therapy for hepatocellular carcinoma. Hepatol Res 2015;45:540-547.

12. Song JH, Son SH, Kay CS, Jang HS. Identification of biologically effective dose-volumetric parameters that predict radiation-induced hepatic toxicity in patients treated with helical tomotherapy for unresectable locally advanced hepatocellular carcinoma. Medicine (Baltimore) 2015;94:e1904.

13. Son SH, Choi BO, Ryu MR, et al. Stereotactic body radiotherapy for patients with unresectable primary hepatocellular carcinoma: dose-volumetric parameters predicting the hepatic complication. Int J Radiat Oncol Biol Phys 2010;78:1073-1080.

14. Liang SX, Huang XB, Zhu XD, et al. Dosimetric predictor identification for radiation-induced liver disease after hypofractionated conformal radiotherapy for primary liver carcinoma patients with Child-Pugh Grade A cirrhosis. Radiother Oncol 2011;98:265-269.

15. Cheng JC, Wu JK, Lee PC, et al. Biologic susceptibility of hepatocellular carcinoma patients treated with radiotherapy to radiation-induced liver disease. Int J Radiat Oncol Biol Phys 2004;60:1502-1509.

16. Cardenes HR, Price TR, Perkins SM, et al. Phase I feasibility trial of stereotactic body radiation therapy for primary hepatocellular carcinoma. Clin Transl Oncol 2010;12:218225.

17. Pan CC, Kavanagh BD, Dawson LA, et al. Radiation-associated liver injury. Int J Radiat Oncol Biol Phys 2010;76(3 Suppl):S94-S100.

18. Liaw YF, Sung JJ, Chow WC, et al. Lamivudine for patients with chronic hepatitis B and advanced liver disease. $\mathrm{N}$ Engl J Med 2004;351:1521-1531.

19. Guha C, Kavanagh BD. Hepatic radiation toxicity: avoidance and amelioration. Semin Radiat Oncol 2011;21:256263.

20. Scorsetti M, Comito T, Cozzi L, et al. The challenge of inoperable hepatocellular carcinoma (HCC): results of a single-institutional experience on stereotactic body radiation therapy (SBRT). J Cancer Res Clin Oncol 2015;141:13011309.

21. Mendez Romero A, Wunderink W, Hussain SM, et al. Stereotactic body radiation therapy for primary and metastatic liver tumors: a single institution phase I-II study. Acta Oncol 2006;45:831-837.

22. Okajima C, Arii S, Tanaka S, et al. Prognostic role of ChildPugh score 5 and 6 in hepatocellular carcinoma patients 
who underwent curative hepatic resection. Am J Surg 2015;209:199-205.

23. Kim HY, Park JW, Joo J, et al. Worse outcome of sorafenib therapy associated with ascites and Child-Pugh score in advanced hepatocellular carcinoma. J Gastroenterol Hepatol 2013;28:1756-1761.

24. Huertas A, Baumann AS, Saunier-Kubs F, et al. Stereotactic body radiation therapy as an ablative treatment for inoperable hepatocellular carcinoma. Radiother Oncol 2015;115:211-216.

25. Andolino DL, Johnson CS, Maluccio M, et al. Stereotactic body radiotherapy for primary hepatocellular carcinoma. Int J Radiat Oncol Biol Phys 2011;81:e447-e453.

26. Tse RV, Hawkins M, Lockwood G, et al. Phase I study of individualized stereotactic body radiotherapy for hepato- cellular carcinoma and intrahepatic cholangiocarcinoma. J Clin Oncol 2008;26:657-664.

27. Kim TH, Kim DY, Park JW, et al. Dose-volumetric parameters predicting radiation-induced hepatic toxicity in unresectable hepatocellular carcinoma patients treated with three-dimensional conformal radiotherapy. Int J Radiat Oncol Biol Phys 2007;67:225-231.

28. Son SH, Jang HS, Jo IY, et al. Significance of an increase in the Child-Pugh score after radiotherapy in patients with unresectable hepatocellular carcinoma. Radiat Oncol 2014;9:101.

29. Culleton S, Jiang H, Haddad CR, et al. Outcomes following definitive stereotactic body radiotherapy for patients with Child-Pugh B or C hepatocellular carcinoma. Radiother Oncol 2014;111:412-417. 